\title{
Operational Characteristics of a Low-Energy FARAD Thruster
}

\author{
Kurt A. Polzin* \\ NASA-Marshall Space Flight Center, Huntsville, AL 35812
}

\author{
M. Frank Rose ${ }^{\dagger}$ and Robert Miller ${ }^{\ddagger}$ \\ Radiance Technologies, Huntsville, AL 35805
}

\begin{abstract}
Data from a $100 \mathrm{~J}$ per pulse electrodeless accelerator employing pulsed RF-preionization are presented to gain insight into the accelerator's operating characteristics. The data suggest that the propellant distribution is highly unoptimized, with most of the gas inaccessible to the discharge and the remainder mostly concentrated at the inner radius of the coil. The pulsed RF-preionization discharge produces a visible plasma, but like the gas distribution it mostly appears concentrated at the inner radius of the thruster. Magnetic field probes in the discharge point to a current sheet that is not magnetically impermeable. These data also exhibit signs of nonrepeatability, and time-integrated discharge photography shows signs of spatial nonuniformity in both the radial and azimuthal directions. Terminal voltage measurements on the two capacitor banks of the thruster do not exhibit the asymmetric nature (in time) typically associated with an efficient pulsed plasma accelerator. Based on the experimental evidence, the poor performance of the thruster is thought to be due to insufficient preionization, which at these low discharge energy levels severely limits the ability of the main current pulse to couple with and effectively accelerate the propellant.
\end{abstract}

\section{Introduction}

$\mathrm{T}$ HE Faraday Accelerator with Radio-frequency Assisted Discharge (FARAD) ${ }^{1}$ is a type of pulsed inductive plasma accelerator being developed for spacecraft propulsion. The device is electrodeless, with capacitively-stored energy discharged through an inductive coil to induce a plasma near the coil face. The plasma is expelled at high exhaust velocity $[\mathcal{O}(10 \mathrm{~km} / \mathrm{s})]$ by the Lorentz body force arising from the interaction of the plasma current and the induced magnetic field.

A $100 \mathrm{~J} /$ pulse laboratory-model FARAD thruster was presented in detail in a supporting companion publication. ${ }^{2}$ The thruster subsystems (mass injection, preionization, and acceleration) are integrated into a single unit using a modular design approach that makes it possible to alter or redesign certain aspects of the unit (shown in Fig. 1 in the present configuration) without necessitating refabrication of the entire thruster. In this paper, the thruster's operation is demonstrated and specific performance parameters are characterized through a series of plasma measurements and discharge photography.

The outline for the rest of this paper is as follows. The diagnostics used to perform measurements on the thruster are presented in Sect. II. The thruster's operating conditions for the data presented in this paper are described in Sect. III. In Sect. IV, data obtained from the thruster are presented, with a discussion of the data following in Sect. V.

\section{Diagnostics}

We proceed with a description of the various measurement devices used in the present work.

\section{A. Voltage Probes}

Measurement of the time-varying voltage on the capacitor bank is performed using a Tektronix P6015A high-voltage probe. This probe is frequency compensated, having a bandwidth of $75 \mathrm{MHz}$. Its output is attenuated by a factor of 1000 , yielding a measure of the capacitor voltage that can be displayed and recorded by a fast oscilloscope.

\footnotetext{
* Propulsion Research Engineer, Propulsion Research and Technology Applications Branch, Propulsion Systems Department. Senior Member AIAA.

$\dagger$ V.P. of Research, Auburn Office, 231 Leach Science Center, Auburn University, Auburn, AL 36849. Associate Fellow AIAA.

${ }^{\ddagger}$ Project Manager, Auburn Office, 231 Leach Science Center, Auburn University, Auburn, AL 36849. Member AIAA.

This material is declared a work of the U.S. Government and is not subject to copyright protection in the United States.
} 


\section{B. Magnetic Field Probes}

The induced magnetic field measurements are acquired using $B$-dot probes. A photo and circuit schematic of the balanced $B$-dot probes used in this study are shown in Fig. 2. The probe heads consist of $220 \mathrm{nH}$ wirewound non-ferrite core Panasonic surface mount inductors, which measure $\mathrm{d} B / \mathrm{d} t$ in the radial $(r)$ and axial $(z)$ directions. The probe head is contained within a glass tube that protects it from the plasma. This tube is inserted into the flow in the axial direction and the tube has a diameter that is on the order of millimeters so as not to significantly disturb the plasma. The numerically integrated $\mathrm{d} B / \mathrm{d} t$ signals are multiplied by their respective calibration constants to obtain $B(t)$ in the $r$ and $z$-directions. The circuit is balanced by connecting the probe heads to the outer taps on 1:1 center-tap transformers using twisted wire pairs while the center taps on the probe side of the transformer are grounded. Circuits balanced in this manner have been shown to offer excellent common-mode signal rejection. ${ }^{3}$ Integration errors dominate this measurement and grow with time, resulting in an overall error at the end of a pulse that is $\sim \pm 5 \%$ of the maximum measured value.

\section{Triple Probes}

Triple probe measurements were attempted using the method described by Chen and Sekiguchi. ${ }^{4}$ Specifically, the current-mode triple probe configuration was used where all three probes are biased relative to each other.

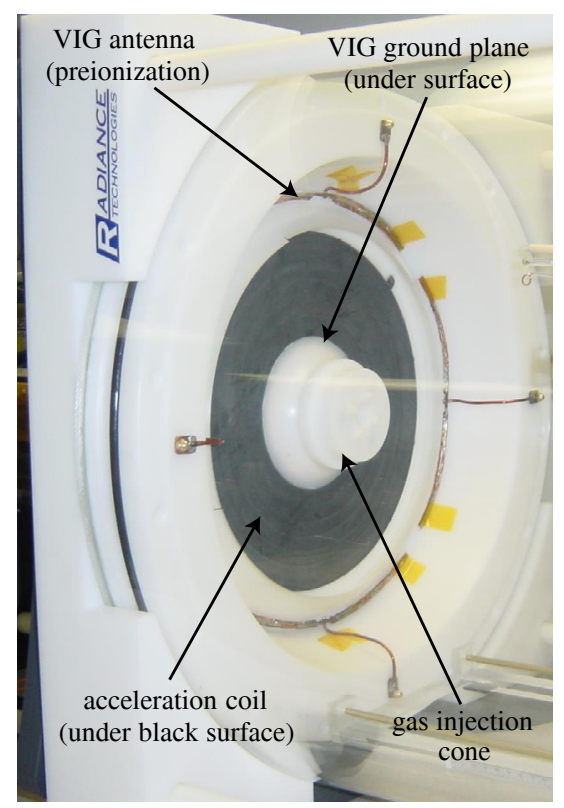

Figure 1. Photograph of the FARAD laboratorymodel thruster coupled to a vacuum chamber. Current levels in the probes were measured using two model 2877 Pearson current monitors, and the voltages on each probe were measured separately relative to ground using 10:1 voltage probes. In our implementation, we encountered significant electrical noise, which was detrimental to obtaining timeresolved measurements of the plasma density or electron temperature. We were able to use the measurements to estimate the overall, average plasma density and temperature.

\section{Discharge Imaging}

Two different cameras were used to obtain images of the plasma discharge. The first was a Canon XL1 video camera which captured images at a rate of 30 frames per second with an exposure time of 1/60 s. The discharge appears as a time-integrated image, and is completely captured in a single frame of video. All images were obtained using an f-stop setting of 16. In addition, a neutral density filter of 1.5 was used in the acquisition of some images. Images of argon-ion emission were captured with this camera using the neutral density filter and a $488 \mathrm{~nm}$ narrow-line filter (10 nm line width).

A second, faster Redlake Motionscope M2 high-speed camera was also used, capturing images at a rate of 1000 frames per second with an exposure time of $1 \mathrm{~ms}$. This camera was used to obtain images of the argon-ion line emission (this time without the neutral density filter). The images are useful in showing the spatial extent of the light emission over the acceleration coil face and in showing that this emission correlates to the presence of a plasma and is not only due to excited, neutral argon light emission.

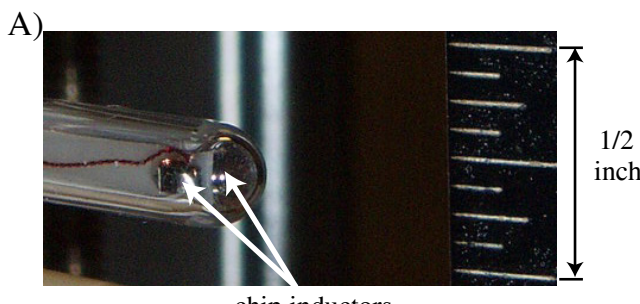

chip inductors
B)

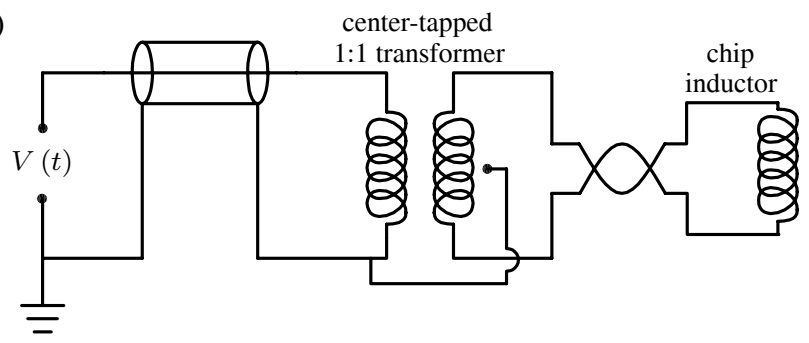

Figure 2. A) Photograph of the $B$-dot probe head. B) Circuit schematic of a balanced $B$-dot probe. 


\section{E. Data Acquisition System}

Various Tektronix two and four channel oscilloscopes were used to acquire the data in our experiments. The scopes were all simultaneously triggered by the pulse that initiated the discharge of the vector inversion generator (VIG). The acquisition rate for the $B$-dot and triple probe data was $250 \mathrm{MS} / \mathrm{sec}$ while the voltages on the capacitor banks were measured at a rate of $125 \mathrm{MS} / \mathrm{sec}$.

\section{Thruster Operating Conditions}

The two capacitor banks in the Bernardes and Merryman (B\&M) powertrain configuration ${ }^{2}$ each have a capacitance of $20 \mu \mathrm{F}$. Unless otherwise noted, a charge voltage of $\sim 3120 \mathrm{~V}$ was used, resulting in an energy per pulse of $100 \mathrm{~J}$. The mass bit to within $\pm 10 \%$ is $150 \mu \mathrm{g} /$ pulse. This was quantified by filling a fixed volume tank with gas, pulsing the valve several times, and measuring the initial and final tank pressure levels. Based on the pressure drop, the average mass bit can be calculated from the ideal gas equation of state. For preionization, the VIG was charged to $2 \mathrm{kV}$. The total energy stored in the VIG at this charge voltage was $0.17 \mathrm{~J}$.

\section{Data}

\section{A. Propellant Injection}

The temporal evolution of the propellant distribution over the acceleration coil face was measured using a fast ionization gauge in the manner described in Ref. [2]. For completeness, we present those results here to show the time-varying neutral propellant distribution over the coil face before the acceleration pulse is initiated. In Ref. [2], it was noted that when the acceleration coil is pulsed at $t=1.0 \mathrm{~ms}, 57 \%$ of the propellant is still in the gas distribution manifold and another 19\% is at an axial distance greater than $3 \mathrm{~cm}$ from the thruster face.
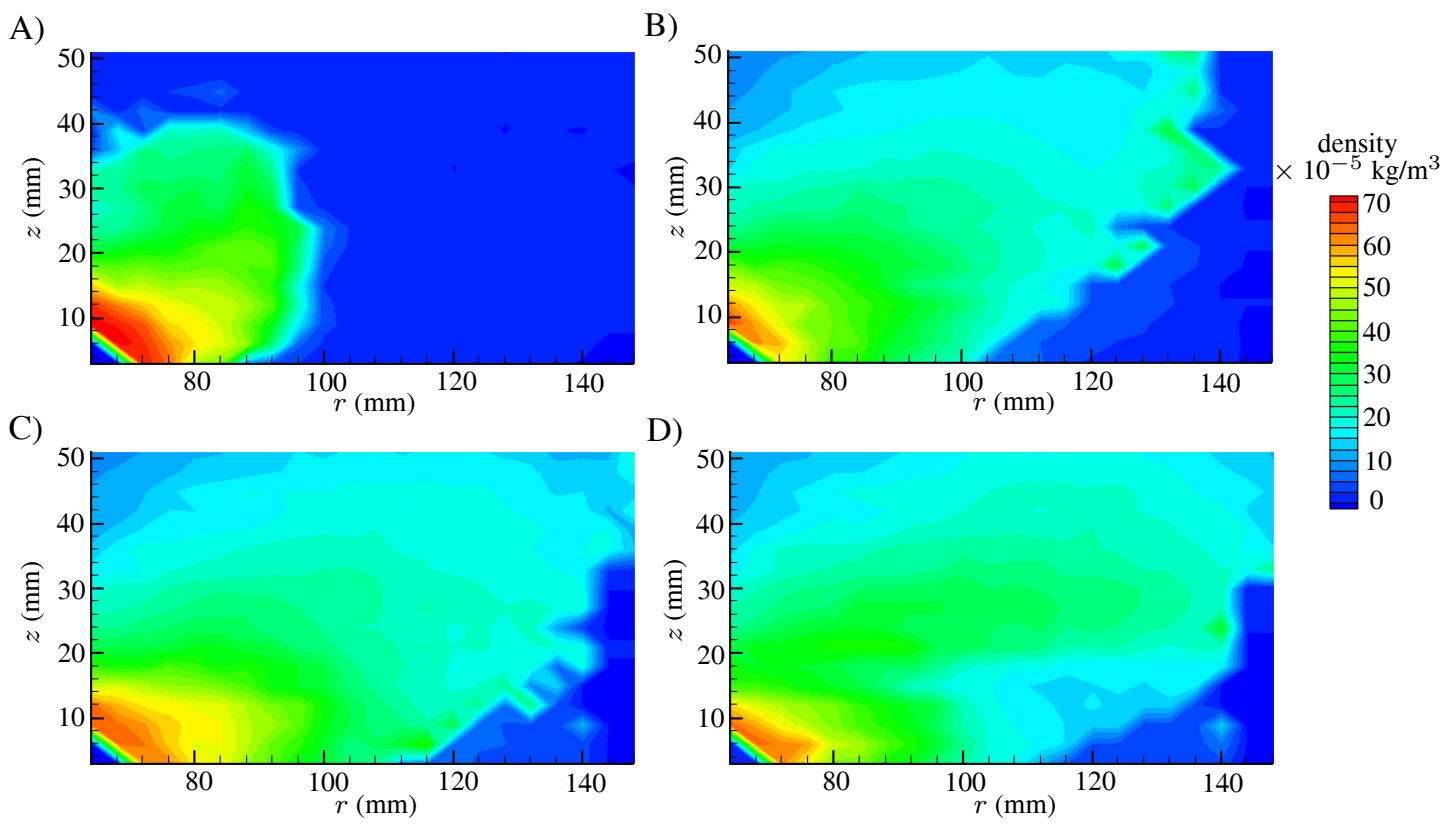

Figure 3. Measured propellant density contours in the thruster at A) $t=0.4 \mathrm{~ms}$, B) $t=0.6 \mathrm{~ms}$, C) $t=0.8 \mathrm{~ms}$, and D) $t=1.0 \mathrm{~ms}$.

\section{B. Circuit Performance}

A circuit schematic showing the B\&M pulsed power train inductively coupled to the plasma is given in Fig. 4A. In this configuration, the capacitor $C_{1}$ is charged to $3120 \mathrm{~V}$ and then discharged when the solid-state switch is closed. The voltages on both capacitors $C_{1}$ and $C_{2}$ are given in Fig. 4B for two separate cases; one where there is a plasma present over the acceleration coil face (solid line) and one where there is not (dashed line). In each 

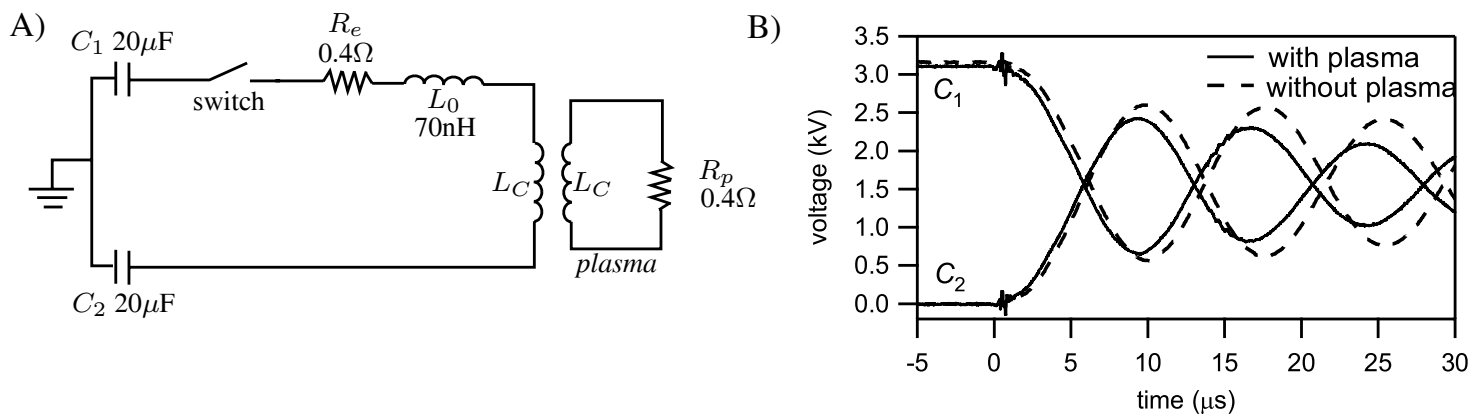

Figure 4. A) Circuit schematic of the B\&M pulse powertrain. B) Time-varying voltages on the capacitors in the B\&M for $100 \mathrm{~J}$ pulses with and without plasma present over the face of the acceleration coil.
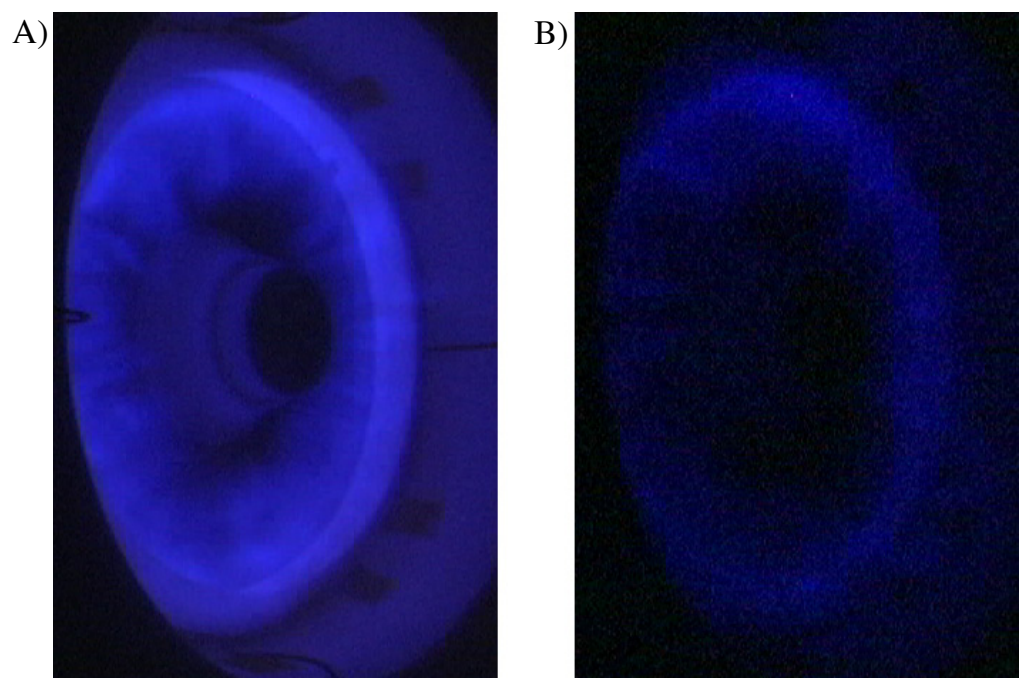

Figure 5. Time-integrated color image of the discharge from a single shot of the FARAD thruster operating at a discharge energy of 100 J captured using a video camera A) through a 1.5 neutral density filter and B) through both a 1.5 neutral density filter and a $488 \mathrm{~nm}$ argon-ion line filter (contrast enhanced +60$)$.

case the voltages oscillate about a voltage half-way between zero and the charge voltage, eventually equilibrating. The discharge period without any plasma present is slightly longer than the period with a plasma present, and the signal attenuates more quickly with a plasma present.

\section{Photographs}

Images of the discharge obtained at a energy of $100 \mathrm{~J}$ per pulse using the Canon XL1 camera are presented in Figs. 5 and 6. The image in Fig. 5A was obtained through a 1.5 neutral density filter and represents the overall light emission from the discharge while the image in Fig. 5B was obtained using both the 1.5 neutral density filter and the $488 \mathrm{~nm}$ argon-ion line filter. The combination of the two filters made the original image very faint. It has been contrast-enhanced +60 in the figure to illustrate the location of emitting argon ions. The total light emission is brightest and appears mostly azimuthally symmetric over the outer one-third of the radial dimension, but some noticeable radial striations appear at smaller radial locations indicat-

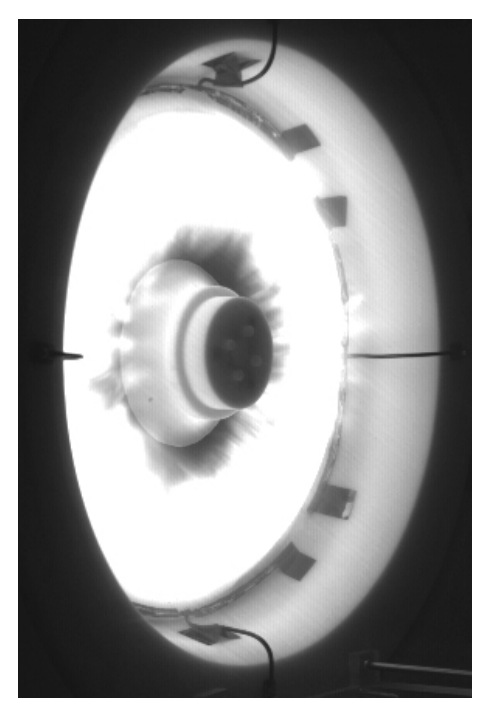

Figure 6. Time-integrated argon ion light emission (488 nm line) from a single shot of the FARAD thruster operating at a discharge energy of $100 \mathrm{~J}$ captured using a 1000 frame per second camera. 

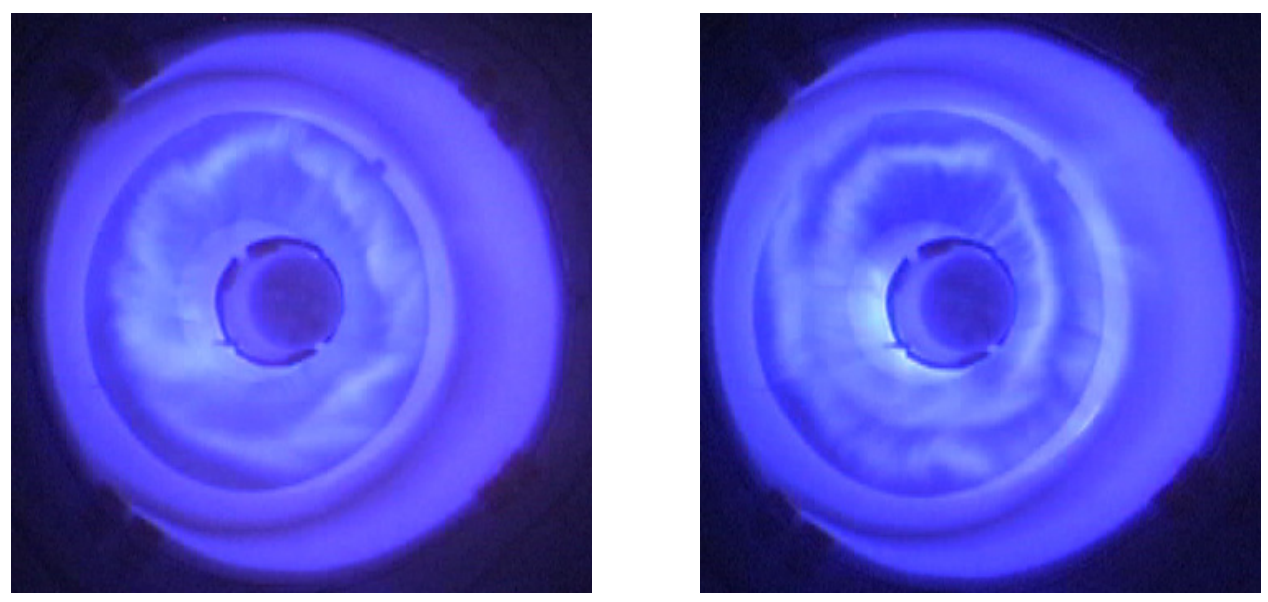

Figure 7. Time-integrated color images of the discharge from two separate pulses of the FARAD thruster operating at a discharge energy of 22.5 J. Images were captured using a video camera.

ing nonuniformity in the discharge. The argon ion light emission is also brightest at larger radii, becoming much more faint with decreasing $r$.

Another argon-ion light emission image at an energy of $100 \mathrm{~J}$ was obtained using the faster Redlake Motionscope M2 camera. This image is presented in Fig. 6. No neutral density filter was used and the image has not been contrastenhanced or processed in any way. The image shows a disk of plasma forming over the acceleration coil except in the middle where there is no coil present under the insulating surface. Striations in the intensity are also visible in this image at the smaller radial locations and they appear to curve in towards the thruster face.

Images of two separate discharges obtained using the Canon XL1 camera at a discharge energy of $22.5 \mathrm{~J}$ (1500 V initial charge voltage) are presented in Fig. 7. The light emission in these instances was much lower than in the 100 $\mathrm{J}$ per pulse case, so no neutral density filter was used. At this lower energy, significant nonuniformities are observed both within a single image and in comparing the two images. This indicates that the current sheet forming over the acceleration coil at this lower energy is nonuniform, nonrepeatable, and poorly organized.

\section{Plasma Measurements}

Data showing the temporal and spatial variation of the radial magnetic field acquired at a radius of $13.5 \mathrm{~cm}$ are presented in Fig. 8. The axial $z$-locations are evenly spaced every $5 \mathrm{~mm}$ between 5 and $40 \mathrm{~mm}$ from the coil face. These data are spliced together over a series of several pulses with the probe moved to a different axial location for each pulse. The induced field reaches a maximum of nearly 1700 gauss at the $z=5 \mathrm{~mm}$ location. Arrows in the figure point to the locations in each waveform where there is an abrupt change in the nature of the profile. These abrupt changes are usually indicative of a current sheet passing a given axial location.

It is observed that even before these changes in the field profiles, there is a considerable magnetic field level reaching the $B$-dot probes at all axial locations. This is characteristic of a fairly weak current sheet that allows a fairly rapid resistive diffusion of the magnetic field produced by the coil.

One property not characterized was the repeatability of these measurements. The striations and nonuniformities in some of the photographs may be indicative of significant nonuniformities in the current sheet, making it difficult to gain insight into the current sheet's motion by way of the spliced magnetic field data presented here.

As previously mentioned the triple probe measurements acquired to measure the number density and electron temperature were very noisy and can only be relied upon here to provide an estimate of either parameter. Given that caveat, the number densities measured were in the range of $1-4 \times 10^{14} \mathrm{~cm}^{-3}$ and the electron temperatures ranges from 1-4 eV. With both parameters, the higher values were found at axial locations that were closer to the coil face and earlier in time.

\section{Discussion}

There are several observations that can be made from the experimental data. The most important is that the current sheet appears very nonuniform, meaning that there is most likely a significant level of shot-to-shot variation in the 

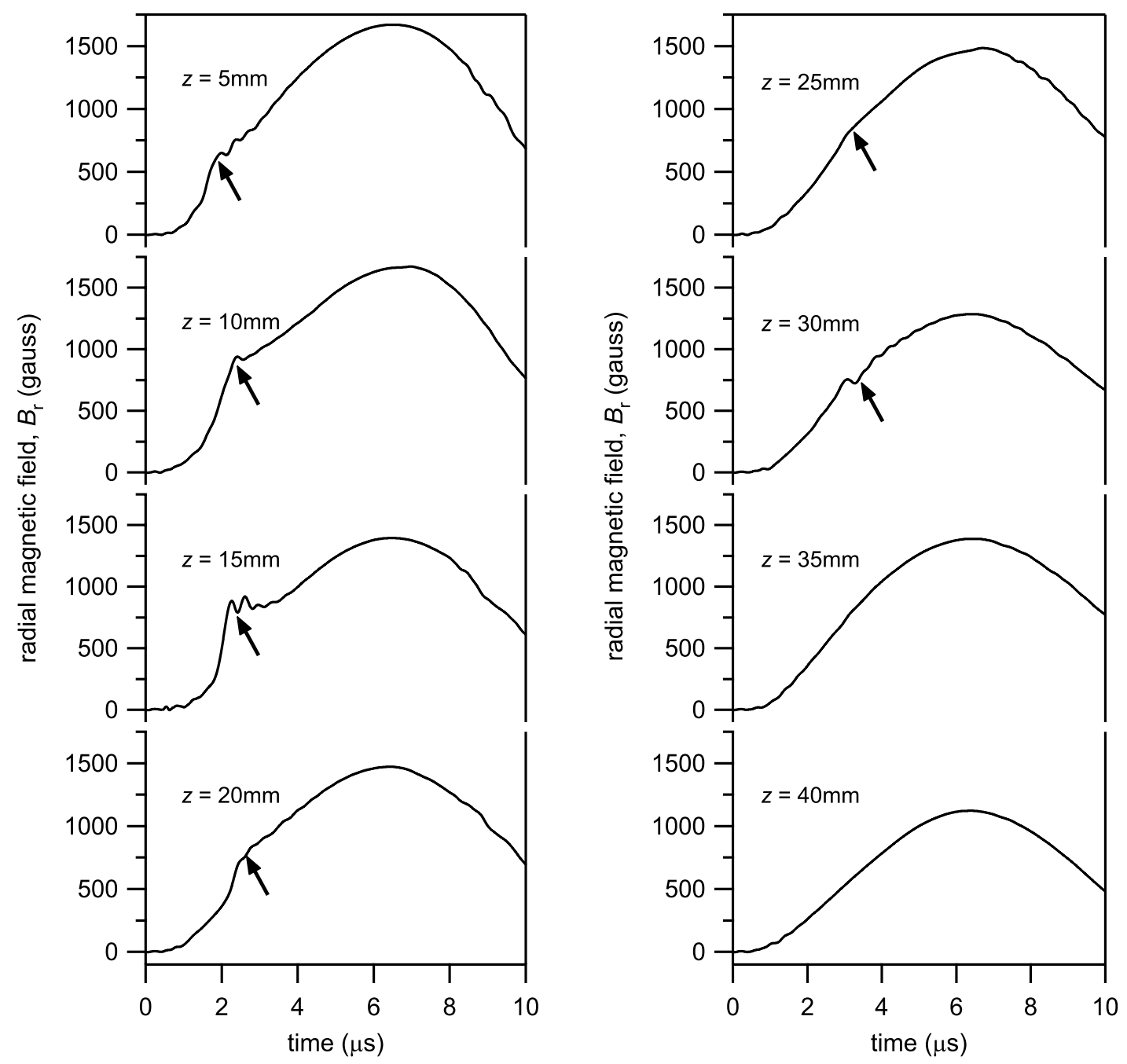

Figure 8. Time histories of the induced radial magnetic field strengths for a discharge energy of $100 \mathrm{~J}$ at various axial stations and at a radius of $13.5 \mathrm{~cm}$. The axial positions are spaced evenly every $5 \mathrm{~mm}$. The arrows point to abrupt changes in the magnetic field profile that are indicative of a current sheet passing the axial location.

measurements. The photographs and the magnetic field measurements both support this finding.

Another observation is that the current sheet does produce an effect on the magnetic field waveforms However, it does not effectively shield downstream locations from the magnetic field produced by the acceleration coil, indicating that it is highly resistive (not magnetically impermeable). The formation of magnetically permeable current sheets has been observed in other pulsed inductive accelerators ${ }^{1,5}$ and the efficiency of these devices suffered as a consequence.

The peak magnetic field produced in the present FARAD experiment is roughly twice the value of the original FARAD proof-of-concept experiment (POCX). ${ }^{1,6}$ A comparison of the data between the two FARAD experiments show that the current sheet produces a greater effect on the magnetic field in the POCX than in the present experiment. Also, comparing the voltage probe data from the present experiment with the current measurements in the POCX, we find that the present experiment has a longer oscillating period.

The voltage measurements on the capacitor banks (Fig. 4B) show that more energy is dissipated when a plasma is present. This is not surprising, but it is not clear whether this energy is dissipated in actual plasma acceleration or in resistive loss processes in the plasma. The other experimental data suggest that the latter case is the more likely conclusion.

As far as either set of data can be trusted, the plasma density in the present experiment is roughly the same order of magnitude as that found in the POCX. ${ }^{1,6}$ The plasma density in the current sheet may be slightly higher in the POCX $\left(7 \times 10^{-14} \mathrm{~cm}^{-3}\right.$ compared with $\left.4 \times 10^{-14} \mathrm{~cm}^{-3}\right)$. 
Differences between the operation of the present experiment and the previous POCX may explain the difficulty in forming consistent, magnetically impermeable current sheets in the former, despite the fact that it operates at a higher discharge energy per pulse (100 J instead of the $78.5 \mathrm{~J}$ in the POCX). The first is the nature of the preionization in each experiment. The present thruster uses a pulsed RF discharge produced by a ringing VIG, while the POCX had a preionized plasma that was directed by an applied magnetic field to the acceleration coil from a $1 \mathrm{~kW}$ steady-state helicon source. The helicon-generated plasma was steady and relatively bright, while the VIG-driven discharge is only visible to the naked eye at the inner radial location where the induced $E$-field is concentrated due to $1 / r$ effects. Given the difference in luminosity between these two cases, it is quite possible that the POCX had a much greater preionized plasma density and that this plasma was much more uniform than that generated by the VIG. Furthermore, it may have been much easier in the POCX for the inductive acceleration coil to effectively couple its energy and form a current sheet at the higher preionized plasma density. ${ }^{\text {a }}$

Another difference between the two FARAD experiments is the nature of propellant injection. The POCX employed a uniform backfill, ensuring a uniform gas density distribution over the acceleration coil face. In the present experiment, gas is injected radially outward, but much of this gas either has not exited the injection manifold or is more than $3 \mathrm{~cm}$ downstream when the current is pulsed. In addition, the current sheet visually appears most dense at larger radii, but Fig. 3 indicates that the gas is most rarified at these locations when the discharge is initiated. As a consequence, there may simply not be enough charge carriers present to form a magnetically impermeable current sheet at these outer locations.

The initial energy and initial voltage in the present experiment are greater than that found in the POCX. Likewise, the measured stray inductance is lower than in the POCX. Despite this, the POCX has a faster current rise rate and rises to a higher peak value, indicating that the stray inductance in the POCX is less than that found in the present accelerator. This could be a result of a combination of differences given above. In the present accelerator, the preionized plasma is weak and does not appear sufficient to effectively couple with the current flowing in the acceleration coil.

Acceleration modeling ${ }^{6,7}$ shows that the thruster is dependent upon the current sheet forming and inductively coupling to the acceleration coil almost immediately after current begins flowing in the coil. The current sheet contains the magnetic flux produced by the coil in the thin region between the coil and the plasma, effectively canceling the coil inductance and permitting the current rise rate to be governed only by the initial voltage and stray inductance in the lines between the capacitor bank and the coil. However, if the current sheet is weak, as is the case in the present accelerator, then the magnetic field rapidly diffuses through the plasma into the open space behind it, significantly increasing the 'stray' inductance presented to the circuit. Increasing the stray inductance in this manner reduces the level of $d I / d t$ in the accelerator, which in turn limits the peak magnetic field strength produced by the coil. Stated another way, a reduction in the level of $d I / d t$ limits the potential to perform electromagnetic acceleration on the plasma. This problem is compounded at the low discharge energies found in the present thruster, where the insufficient preionization can also severely restrict the coupling of energy from the acceleration coil into the current sheet.

\section{Conclusions}

We have presented results from a $100 \mathrm{~J}$ per pulse FARAD laboratory thruster. These experiments were aimed at providing insight into the operation of this accelerator, and many important physical observations can be made from the data:

1. Most of the propellant was inaccessible to the accelerator during the discharge, and the fraction that was available for acceleration was mostly concentrated near the inner radius of the coil.

2. The VIG preionization discharge only produced a visible plasma near the inner radius of the coil.

3. The thruster produced radial magnetic fields close to the acceleration coil that peaked at about 1700 gauss.

4. The thruster failed to produce a magnetically impermeable current sheet, and while the sheet can be observed passing different axial stations, its effects on the magnetic field waveforms are not significant.

5. The current rise rate in the thruster is smaller than in the previous POCX, indicating a higher-than-expected stray inductance in the circuit. The capacitor voltage waveforms appear to support this conclusion, pointing to a highly resistive plasma that does not experience much electromagnetic acceleration.

${ }^{\mathrm{a}}$ It is interesting to note the applied magnetic field in the POCX could be adjusted to significantly reduce the level of preionized plasma reaching the acceleration coil face. Though some fraction of plasma remained over the coil face, a level was reached below which no current sheet was formed. 
6. Photographic evidence shows that there is a plasma present and that this plasma coincides with the location of the overall light emission from the discharge.

7. Spatial nonuniformities are visually observed in the discharge, suggesting that the plasma is not uniform in space and that results are not repeatable from one pulse to another.

8. Insufficient preionization and a very nonuniform propellant distribution lend themselves as leading reasons for the generally poor performance of the thruster. At low discharge energies a weak preionization process, limited by these two effects, can severely limit the ability of the main current pulse to couple with and effectively accelerate the propellant.

\section{Acknowledgments}

This work was performed under NASA contract NNM06AA17G, managed by Dr. Michael LaPointe, supporting the West Virginia High Technology Consortium Foundation's Pulsed Plasma Accelerator (PPA06) program.

\section{References}

\footnotetext{
${ }^{1}$ E.Y. Choueiri and K.A. Polzin, "Faraday Acceleration with Radio-frequency Assisted Discharge," J. Propuls. Power, 22(3):611, May-June 2006.

${ }^{2}$ K.A. Polzin, M.F. Rose, and R. Miller, "Laboratory-Model Integrated-System FARAD Thruster," 44th AIAA/ASME/SAE/ASEE Joint Propuls. Conf., Hartford, CT, July 2008. AIAA Paper 2008-4821.

${ }^{3}$ M. Light, I.D. Sudit, F.F. Chen, and D. Arnush, “Axial Propagation of Helicon Waves,” Phys. Plasmas, 2(11):4094, Nov. 1995.

${ }^{4}$ S.-L. Chen and T. Sekiguchi, "Instantaneous Direct-Display System of Plasma Parameters by Means of Triple Probe," J. Appl. Phys., 36(8):2363, Aug. 1965.

${ }^{5}$ C.L. Dailey and R.H. Lovberg, PIT Clamped Discharge Evolution, Technical Report AFOSR-TR-89-0130, TRW Space and Technology Group, Dec. 1988.

${ }^{6}$ K.A. Polzin, Faraday Accelerator with Radio-frequency Assisted Discharge (FARAD), Ph.D. Thesis, Dept. of Mechanical and Aerospace Engineering, Thesis No. 3147-T, Princeton Univ., Princeton, NJ, 2006.

${ }^{7}$ K.A. Polzin and E.Y. Choueiri, "Performance Optimization Criteria for Pulsed Inductive Plasma Acceleration," IEEE Trans. Plasma Sci., 34(3): $945,2006$.
} 